\title{
Tuning Interfacial Dipole Moment of Spacer Cations for Charge Extraction in Efficient and Ultra-Stable Perovskite Solar Cells
}

Yuankun Qiu, Jianghu Liang, Zhanfei Zhang, Zihao Deng, Heng Xu, Maosheng He, Jianli Wang, Yajuan Yang, Lingti Kong, and Chun-Chao Chen*

School of Materials Science and Engineering, Shanghai Jiao Tong University, Shanghai, 200240, P.R. China

*Corresponding author. c3chen@sjtu.edu.cn

Keywords: dipole moment, built-in electric field, first-principle calculation, perovskite solar cells 

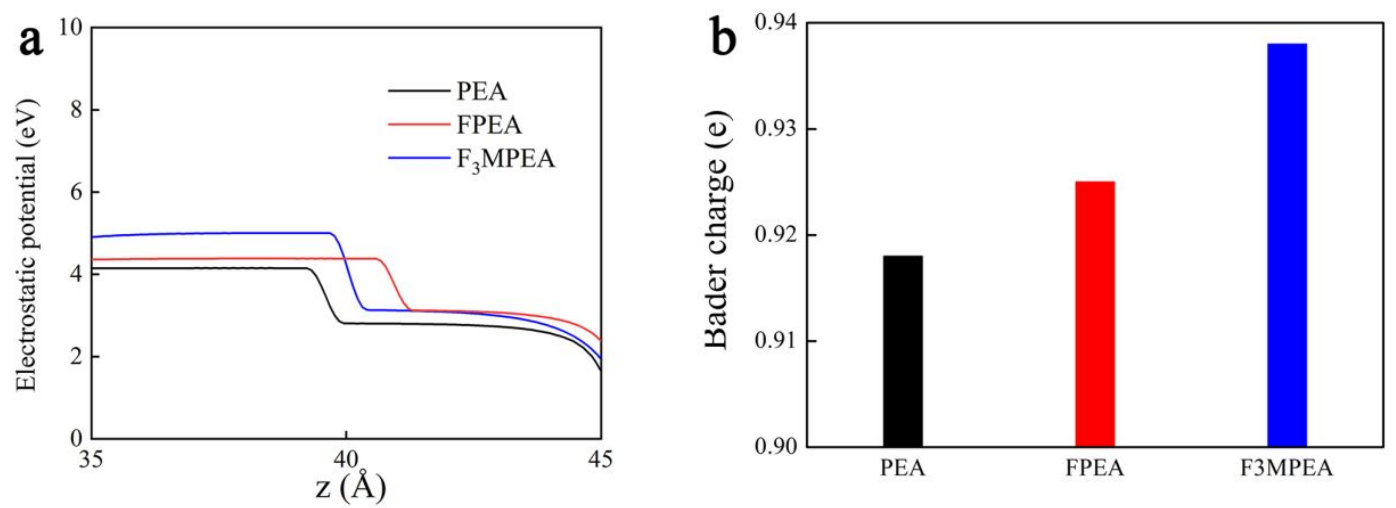

Figure S1. a) Calculated potential steps of 2D/3D heterostructure models. b) Average Bader charge transfer value for 2D/3D heterostructure interface. 

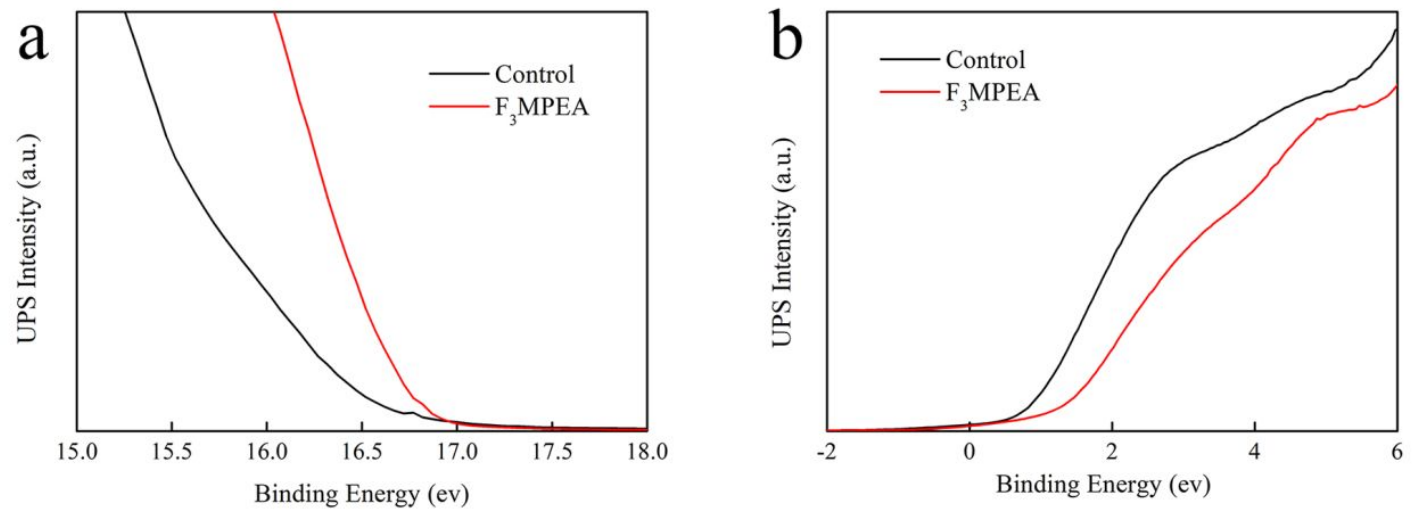

Figure S2. UPS spectra of control and $\mathrm{F}_{3}$ MPEA-based perovskite 


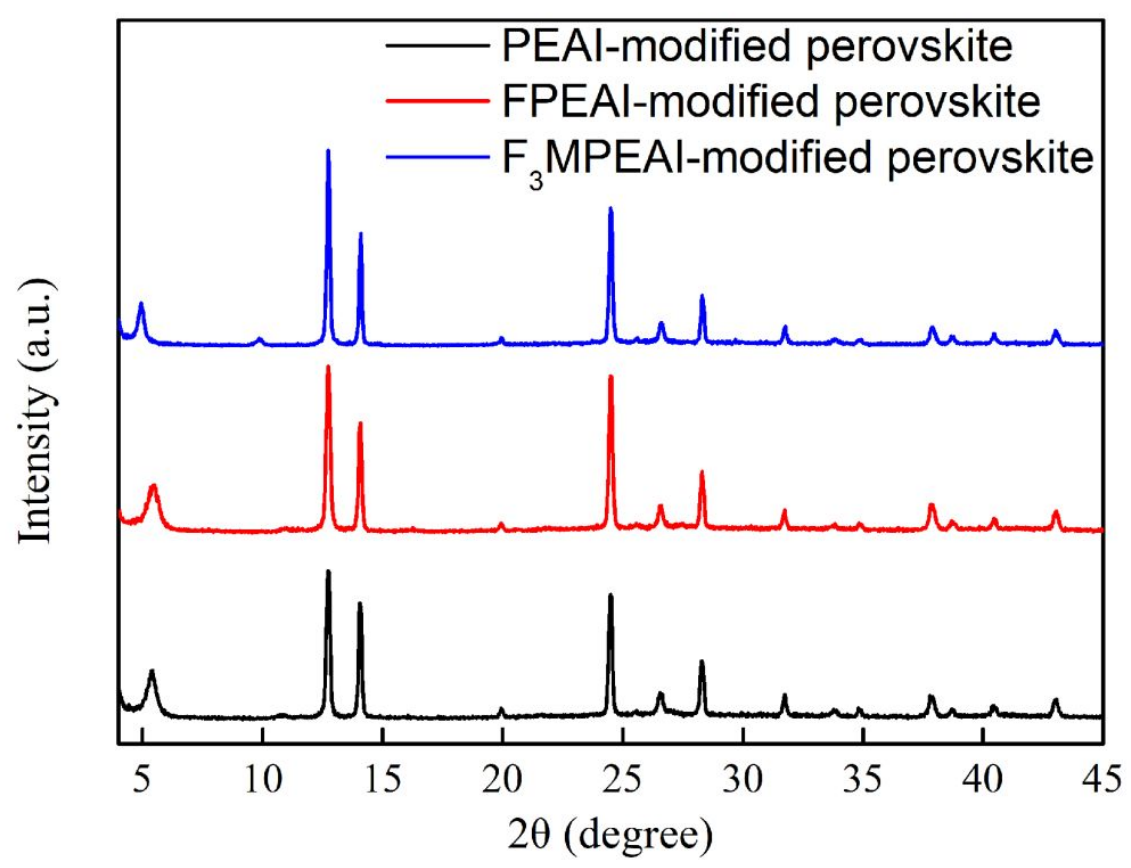

Figure S3 XRD patterns for PEA-, FPEA- and $\mathrm{F}_{3} \mathrm{MPEA}$ - based perovskite films 

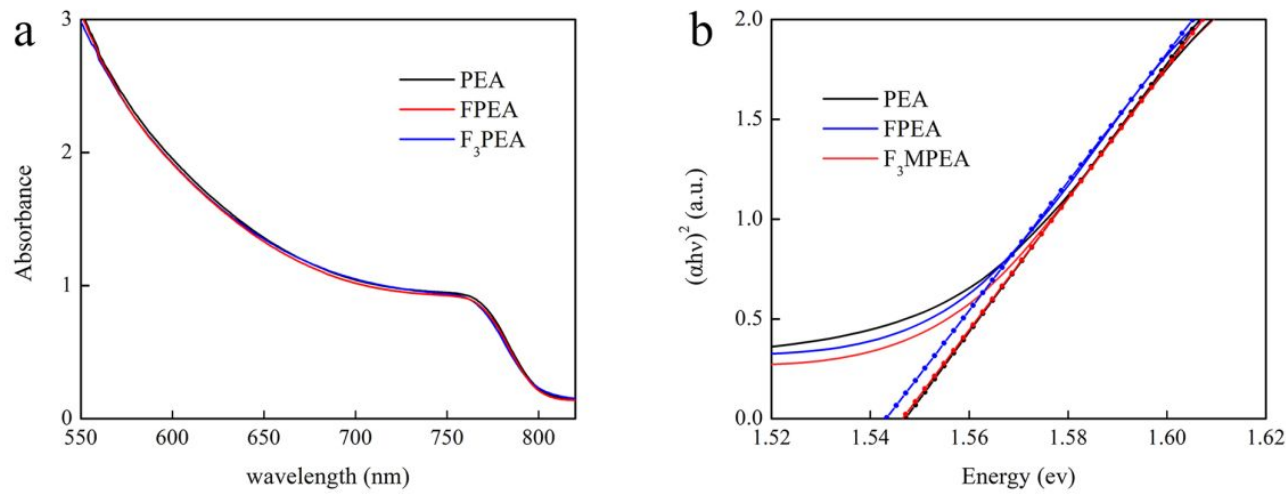

Figure S4. a) absorption spectra for PEA-, FPEA- and $\mathrm{F}_{3}$ MPEA- based perovskite films.

b) Tauc plots derived from absorption spectra. 

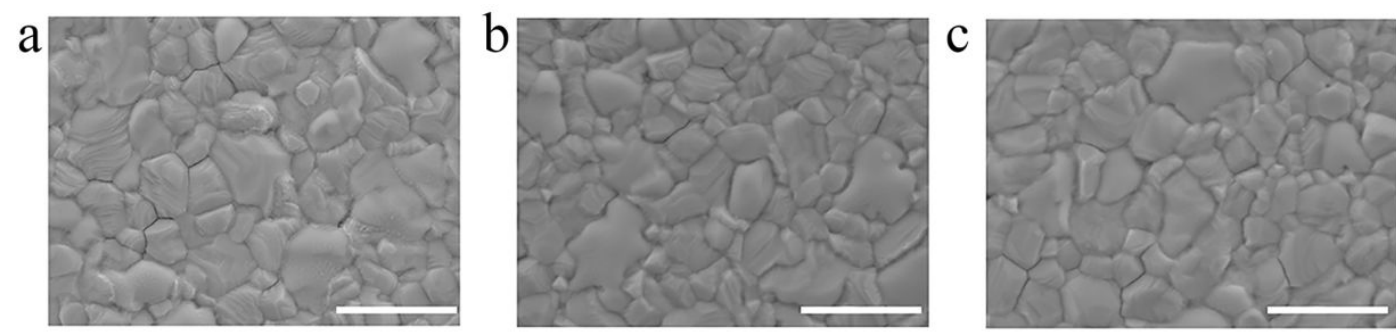

Figure S5. Planar scanning electron microscopy (SEM) images for a) PEA-, b) FPEAand c) $\mathrm{F}_{3}$ MPEA- based 2D/3D perovskite films. 

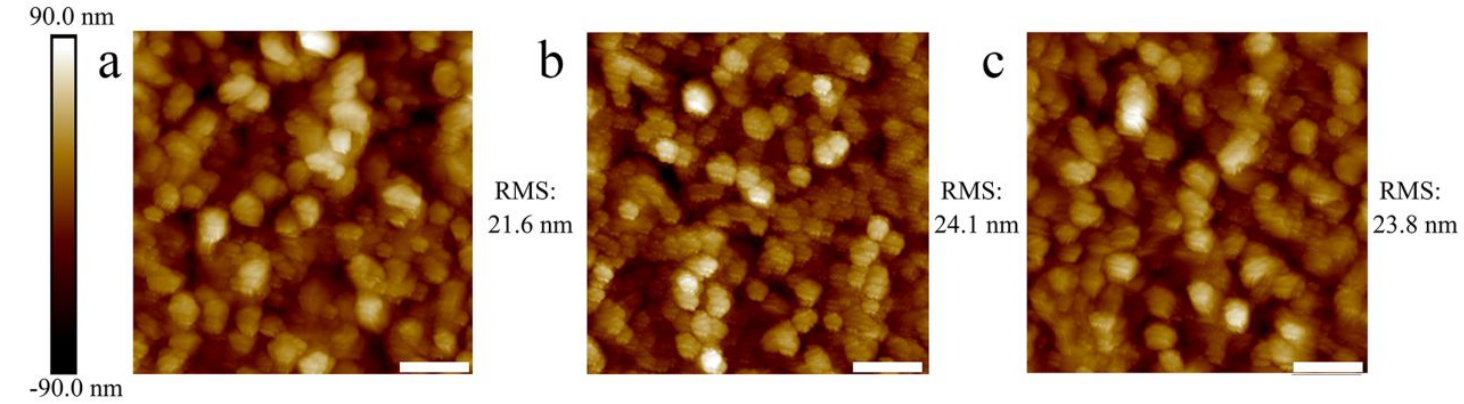

Figure S6 atomic force microscopy (AFM) images for a) PEA-, b) FPEA- and c) $\mathrm{F}_{3}$ MPEA- based 2D/3D perovskite films. 

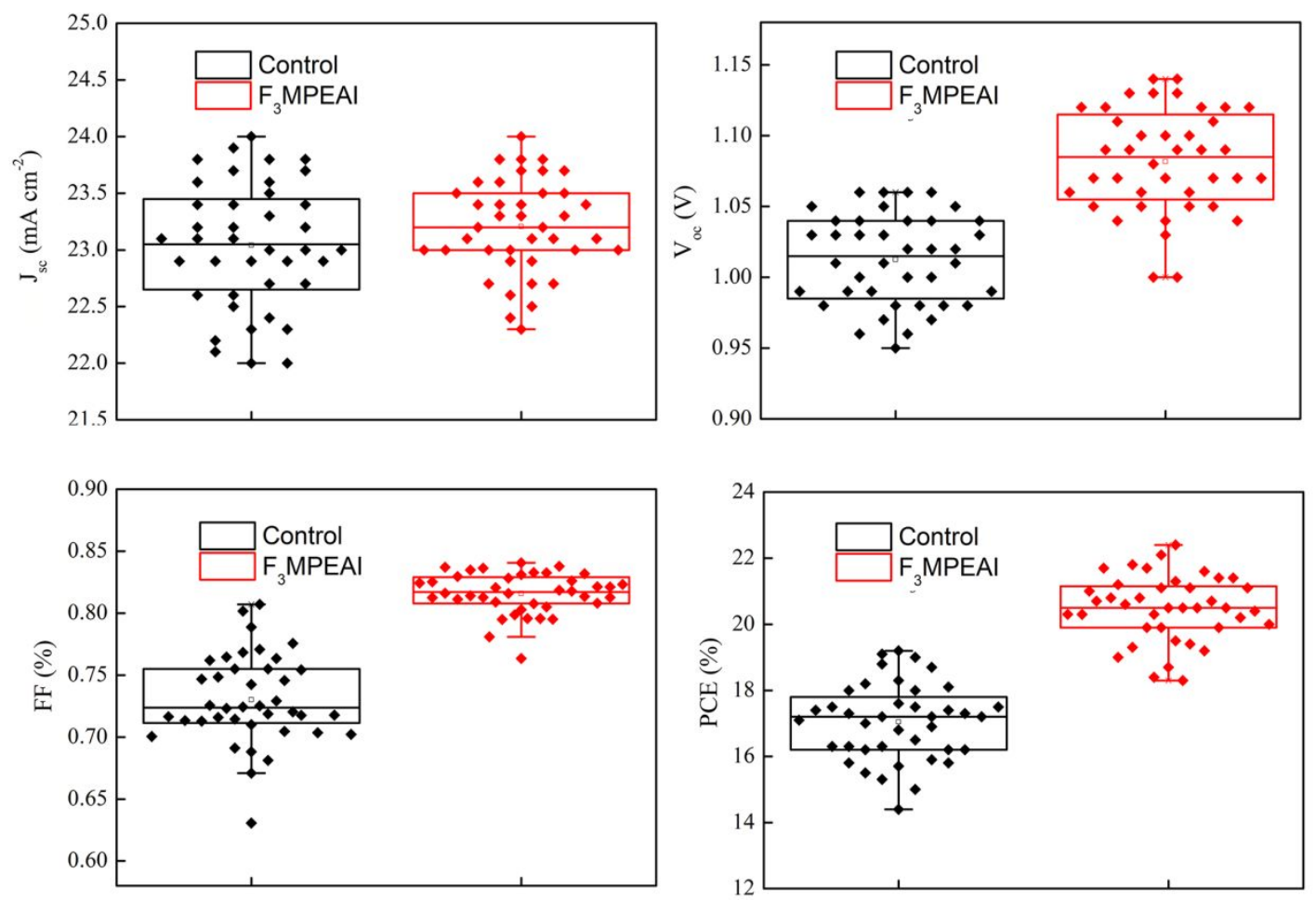

Figure S7. Statistics of $\mathrm{V}_{\mathrm{OC}}, \mathrm{J}_{\mathrm{SC}}, \mathrm{FF}$, and PCE of 40 devices for control and $\mathrm{F}_{3} \mathrm{MPEA}-$ based 2D/3D perovskite. 


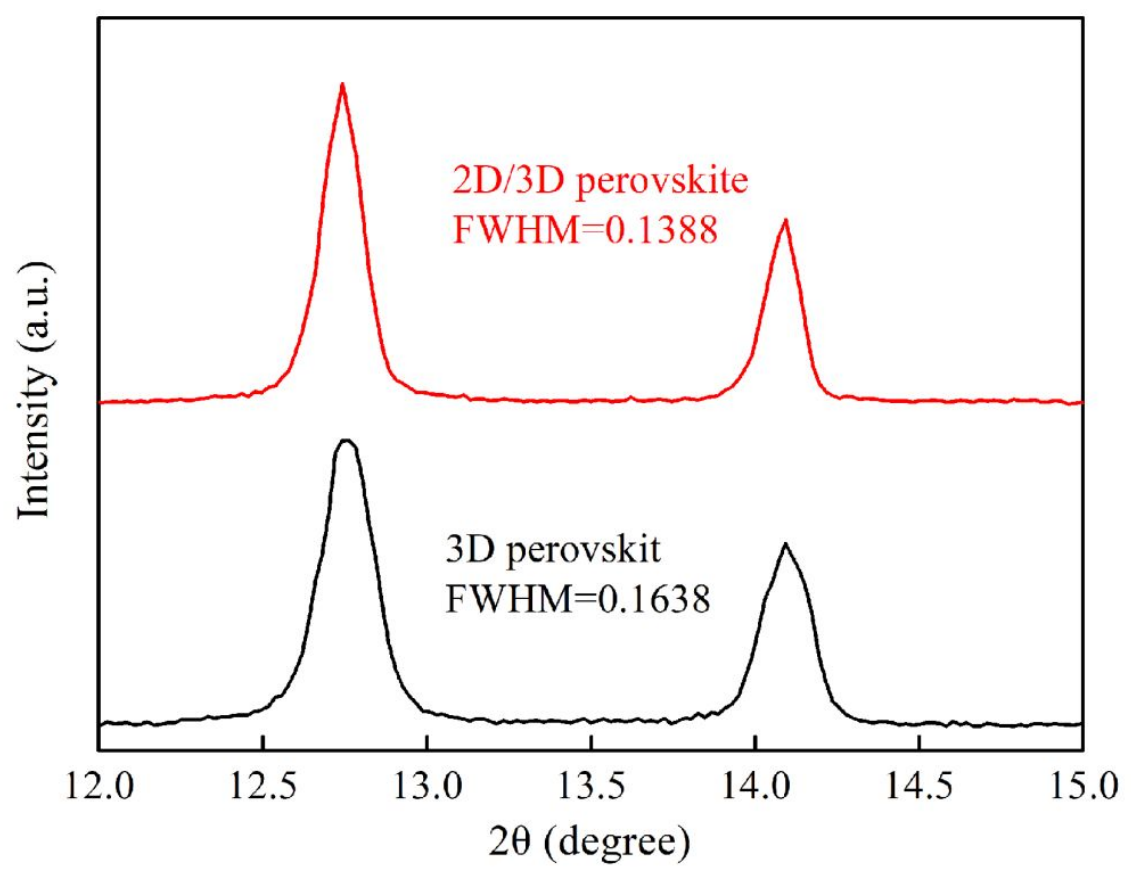

Figure S8. Magnified XRD data of region around $14.0^{\circ}$, Full-width at half-maximum of these corresponding perovskite peaks at $14.1^{\circ}$ were calculated and listed. 
a
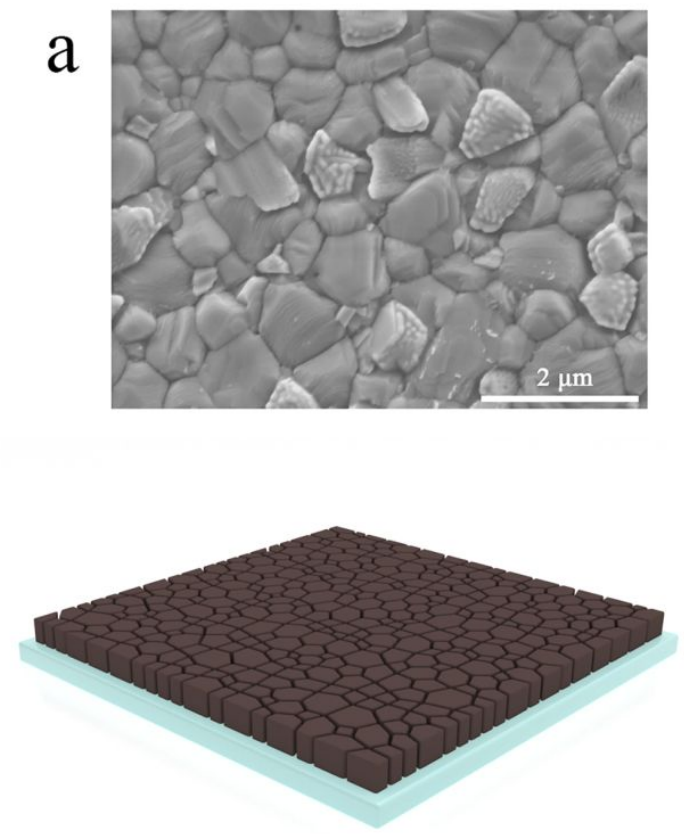

b
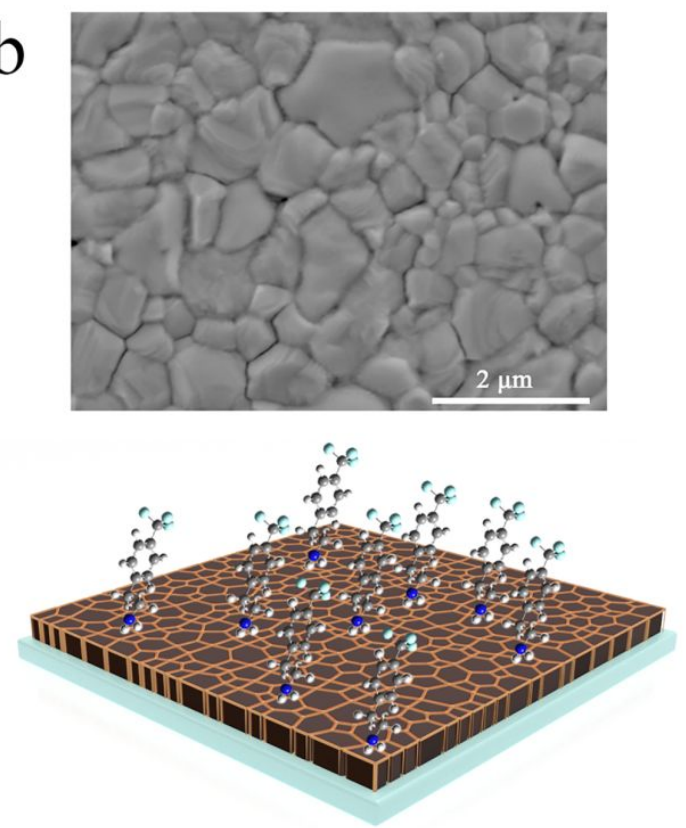

Figure S9. Planar scanning electron microscopy (SEM) images for (a) control and (b)

$\mathrm{F}_{3}$ MPEA-based 2D/3D perovskite films and their schematic illustrations. 


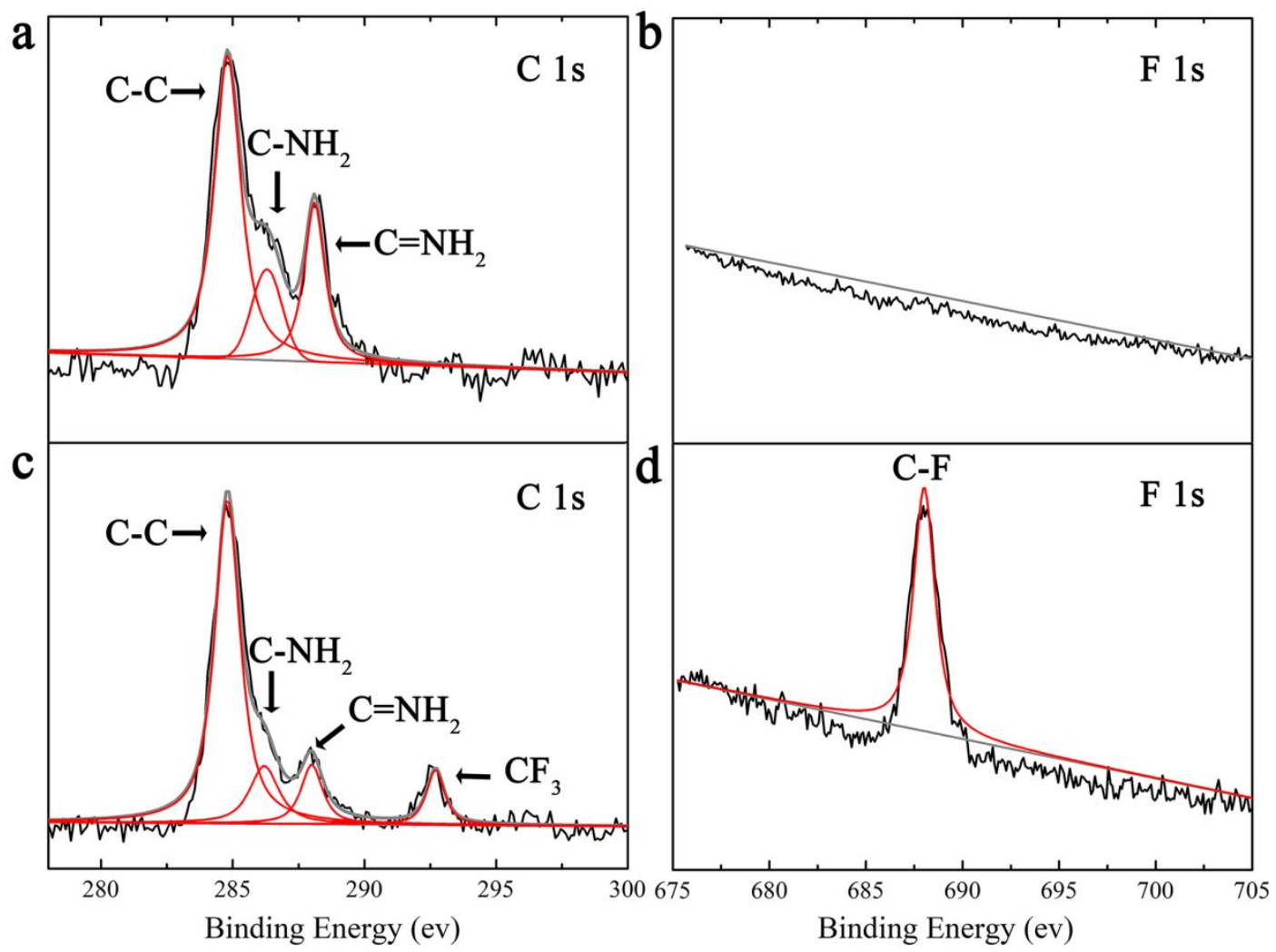

Figure S10. XPS spectra of $C 1$ s and $F 1 s$ for control $(a, b)$ and $F_{3} M P E A-b a s e d ~(c, d)$ perovskite films. 
a

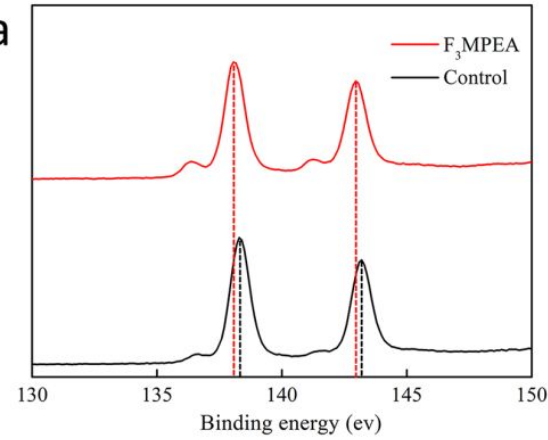

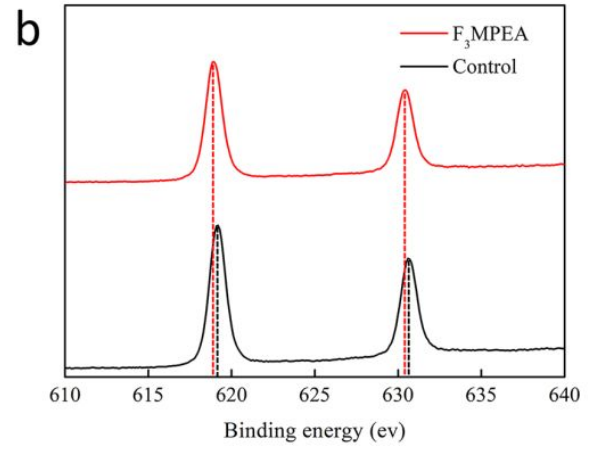

Figure S11. XPS spectra of (a) $\mathrm{Pb} 4 \mathrm{f}$ and (b) I $3 \mathrm{~d}$ for control and $\mathrm{F}_{3} \mathrm{MPEA}$ - based perovskite films. 

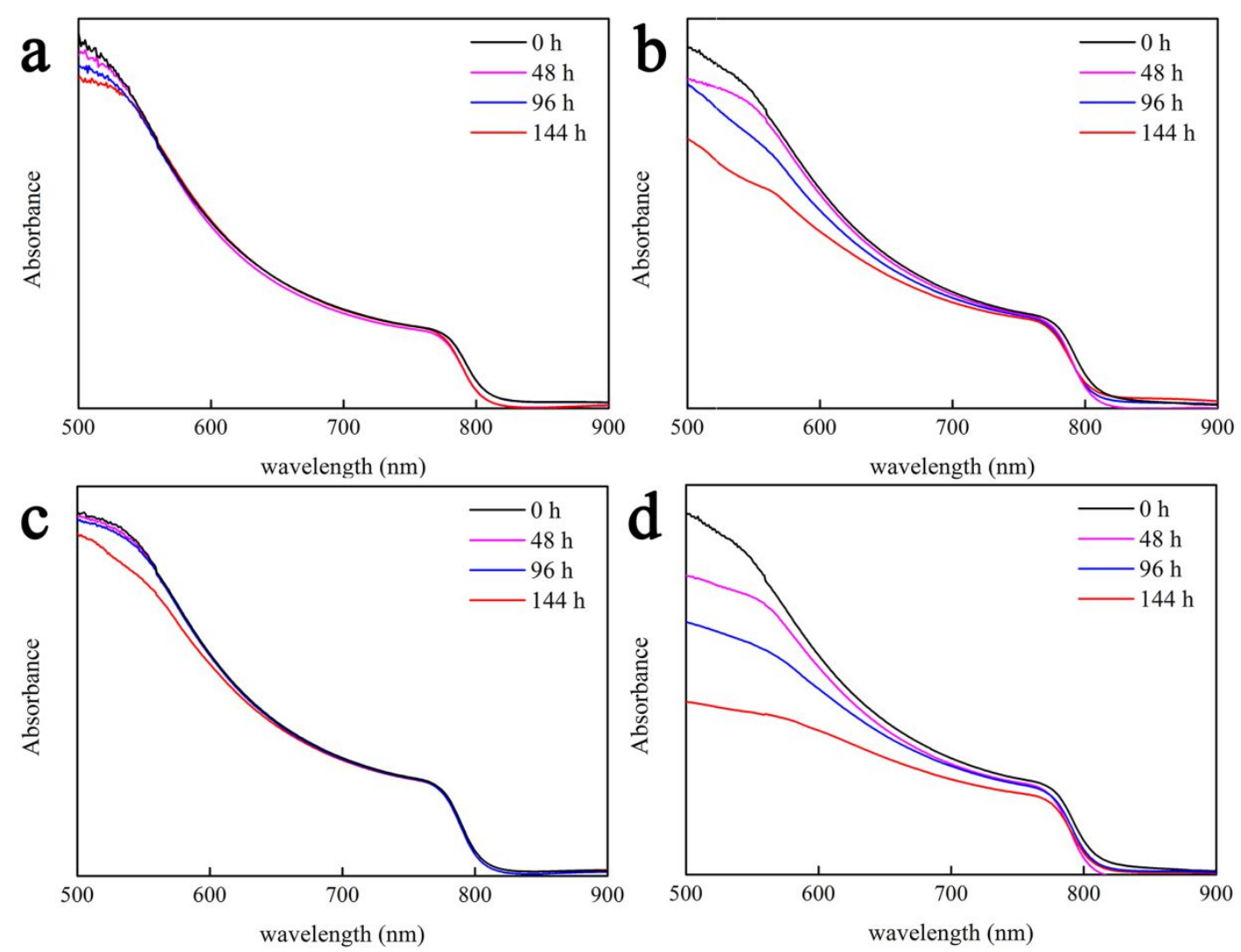

Figure S12. UV-vis absorption spectroscopy for (a) 2D/3D perovskite, (b) 3D perovskite aged at $85^{\circ} \mathrm{C}$ in $\mathrm{N}_{2}$ atmosphere. UV-vis absorption spectroscopy for (c)2D/3D perovskite, (d) 3D perovskite aged at room temperature with an $85 \%$ relative humidity atmosphere. 

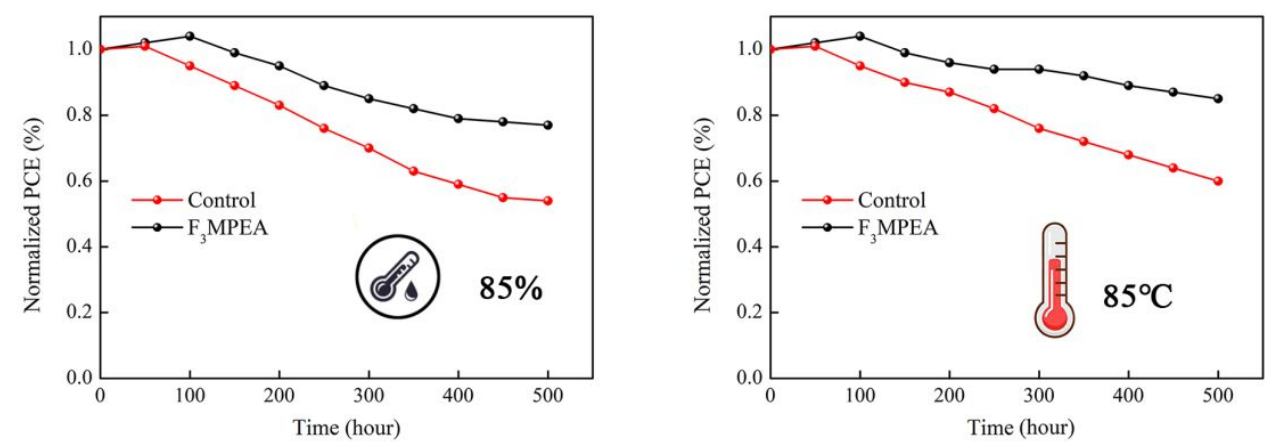

Figure S13. Operational stability of control and $\mathrm{F}_{3} \mathrm{MPEA}$-based devices under $85 \%$ relative humidity or under $85^{\circ} \mathrm{C}$ for 100 hours. 

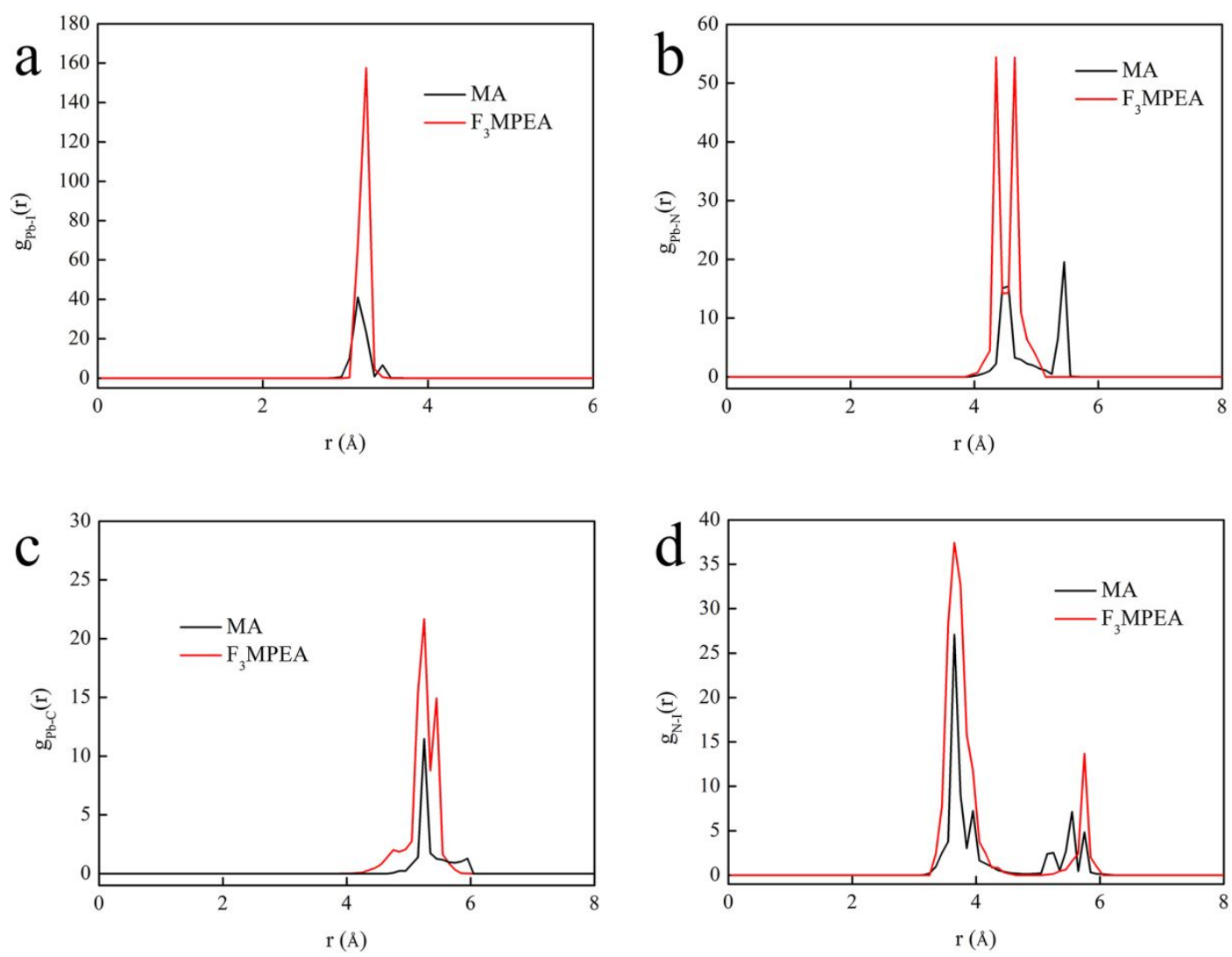

Figure S14. RDF of (a) $\mathrm{Pb}-\mathrm{I}$, (b) $\mathrm{Pb}-\mathrm{N}$, (c) $\mathrm{Pb}-\mathrm{C}$ and (d) N-I bonds in two structures derived from ab initio molecular dynamics (AIMD) results. 


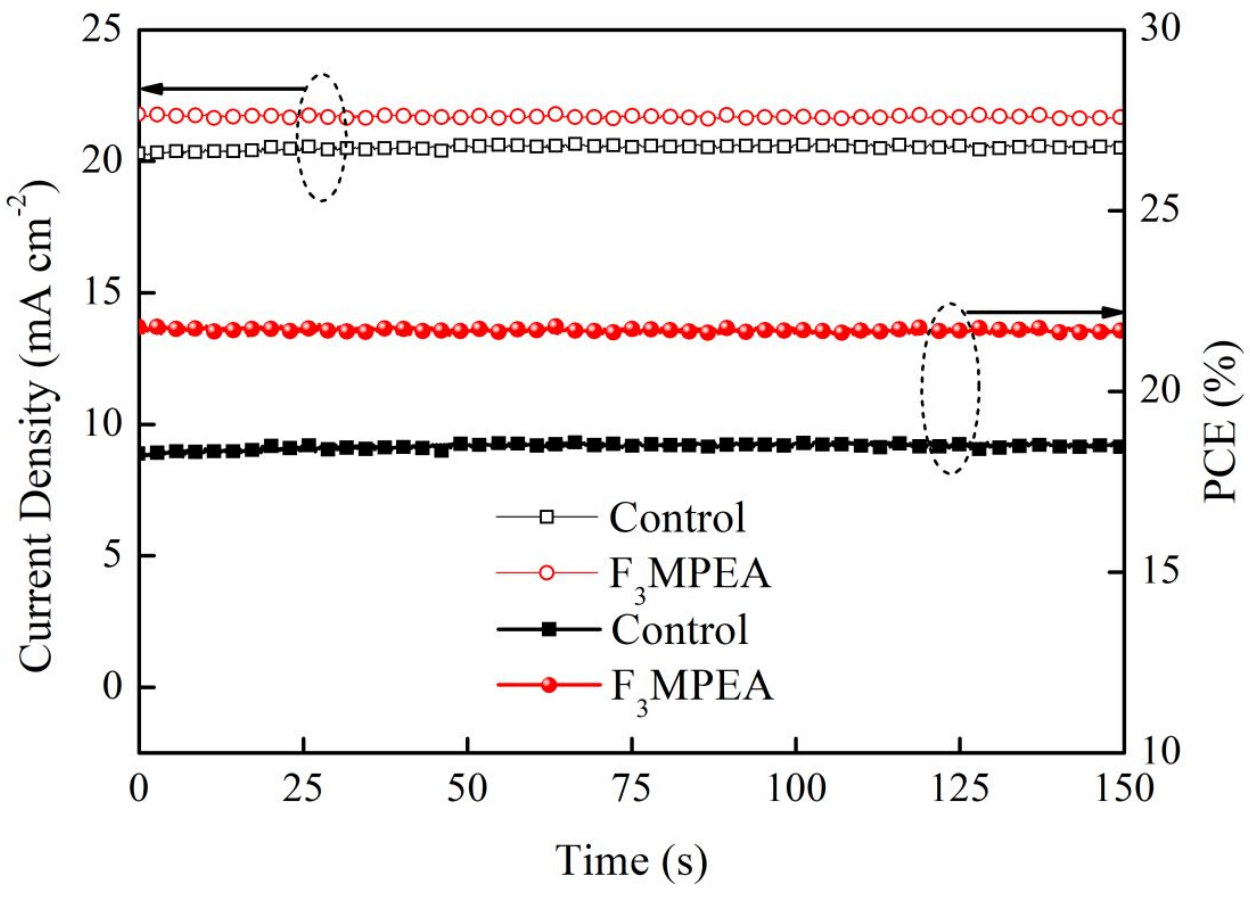

Figure S15. Stabilized current density and PCEs at the maximum power points ( $\mathrm{F}_{3}$ MPEA: $1.0 \mathrm{~V}$; control: $0.9 \mathrm{~V}$ ) of $\mathrm{F}_{3}$ MPEA-based and control devices. 

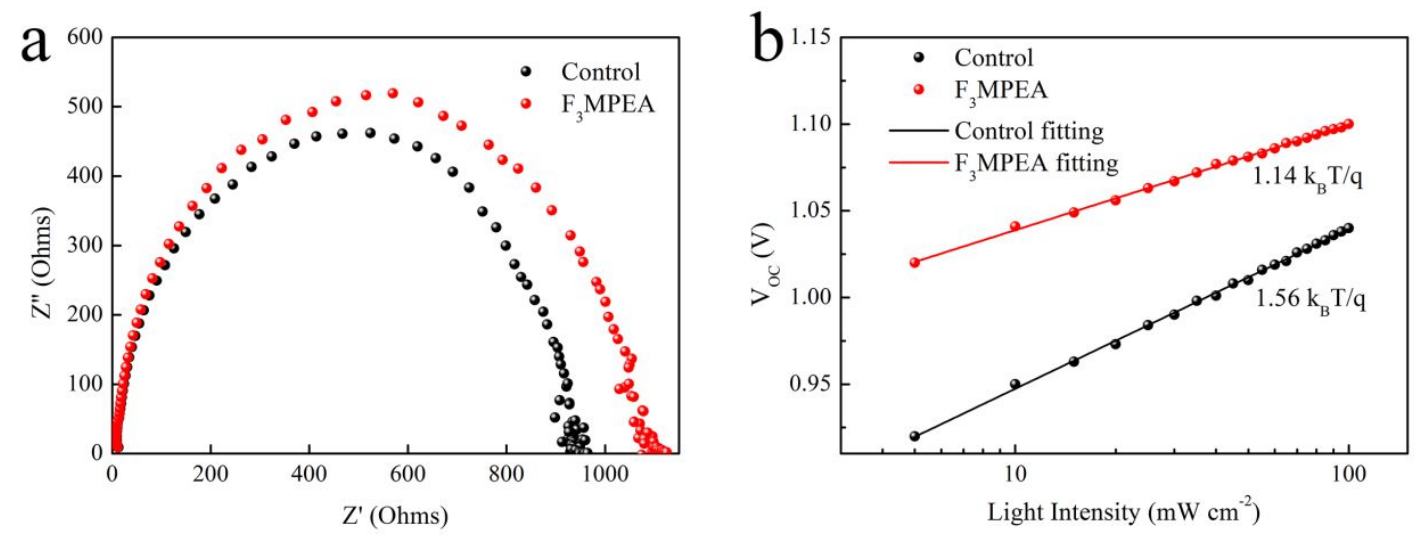

Figure S16. a) Electrochemical impedance spectroscopy of $\mathrm{F}_{3} \mathrm{MPEA}-$ based 2D/3D devices and control devices. b) $\mathrm{V}_{\mathrm{OC}}$ versus light intensity and fitting curves for $\mathrm{F}_{3}$ MPEA-based 2D/3D devices and control devices. 

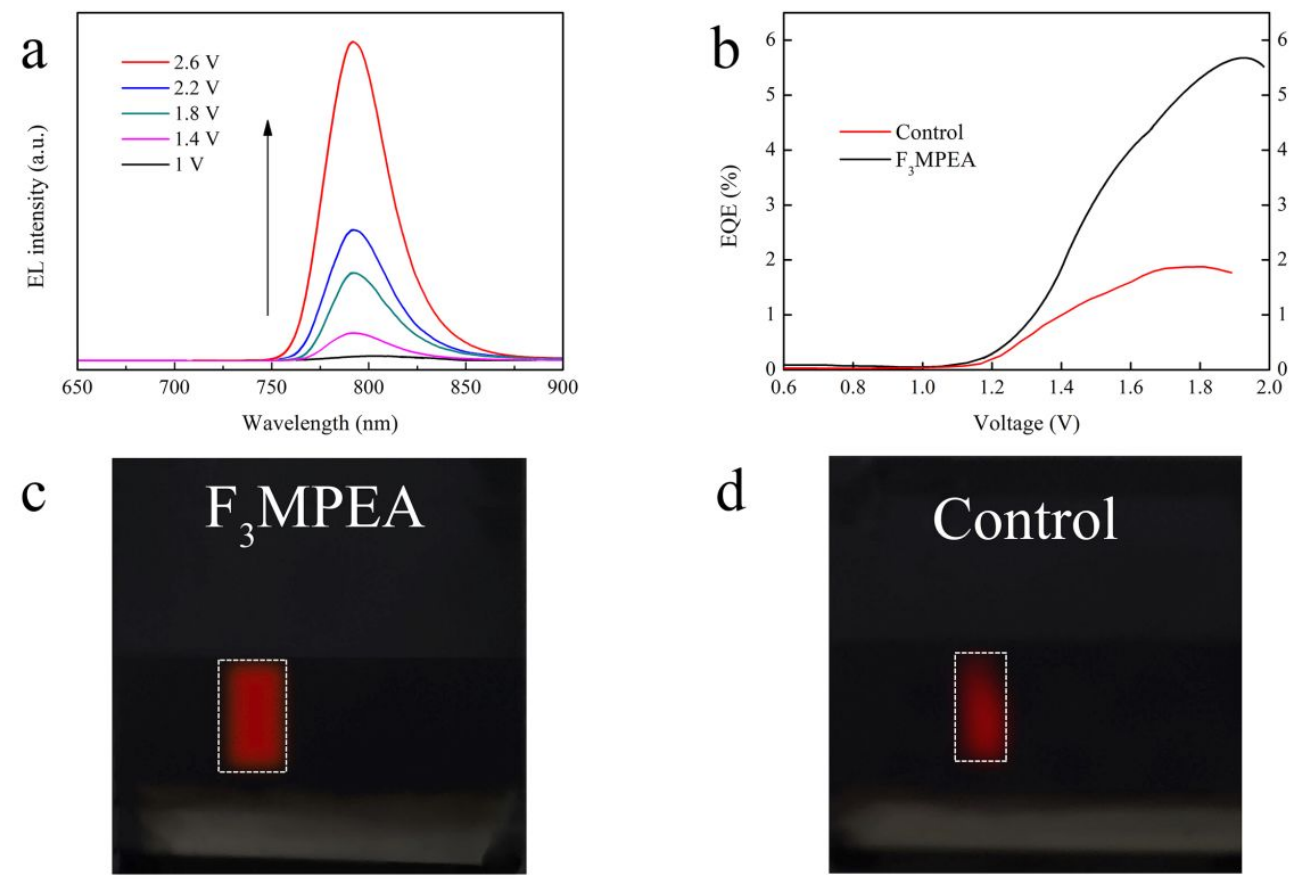

Figure S17. a) Electroluminescence spectra of $\mathrm{F}_{3} \mathrm{MPEA}$-based 2D/3D devices under different voltage bias. b) EL EQE as a function of voltage for $\mathrm{F}_{3} \mathrm{MPEA} 2 \mathrm{D} / 3 \mathrm{D}$ devices and 3D control devices. c, d) Photograph of $\mathrm{F}_{3}$ MPEA-based 2D/3D devices and 3D control devices operating as a LED. 


\begin{tabular}{clccccc}
\hline $\begin{array}{c}\text { Perovskite } \\
\text { components }\end{array}$ & $\mathrm{a} / \AA$ & $\mathrm{b} / \AA$ & $\mathrm{c} / \AA$ & $\alpha /{ }^{\circ}$ & $\beta /{ }^{\circ}$ & $\gamma^{\circ}$ \\
\hline$(\mathrm{PEA})_{2} \mathrm{PbI}_{4}$ & 8.69575 & 9.07378 & 16.61342 & 92.8824 & 86.7176 & 90.0596 \\
$(\mathrm{FPEA})_{2} \mathrm{PbI}_{4}$ & 8.65937 & 9.10265 & 16.15102 & 90.4996 & 95.1932 & 90.0115 \\
$\left(\mathrm{~F}_{3} \mathrm{MPEA}\right)_{2} \mathrm{PbI}_{4}$ & 8.47831 & 9.06933 & 18.12635 & 90.0480 & 103.5634 & 89.6765 \\
$(\mathrm{PEA})_{2} \mathrm{MAPb}_{2} \mathrm{I}_{7}$ & 8.67454 & 8.69594 & 23.97532 & 89.1522 & 87.6423 & 90.0119 \\
$(\mathrm{FPEA})_{2} \mathrm{MAPb}_{2} \mathrm{I}_{7}$ & 8.75557 & 9.01534 & 22.18671 & 89.8729 & 96.0538 & 90.0137 \\
$\left(\mathrm{~F}_{3} \mathrm{MPEA}\right)_{2} \mathrm{MAPb}_{2} \mathrm{I}_{7}$ & 8.67561 & 8.91247 & 26.05539 & 87.2559 & 93.9363 & 89.7107 \\
$(\mathrm{PEA})_{2} \mathrm{MA}_{2} \mathrm{~Pb}_{3} \mathrm{PbI}_{10}$ & 8.68988 & 8.81071 & 30.86041 & 89.5120 & 85.9499 & 89.9839 \\
$(\mathrm{FPEA})_{2} \mathrm{MA}_{2} \mathrm{~Pb}_{3} \mathrm{PbI}_{10}$ & 8.75372 & 8.97184 & 28.90280 & 89.6703 & 95.0639 & 89.9687 \\
$\left(\mathrm{~F}_{3} \mathrm{MPEA}\right)_{2} \mathrm{MA}_{2} \mathrm{~Pb}_{3} \mathrm{PbI}_{10}$ & 8.67828 & 8.89937 & 32.93851 & 87.7716 & 92.3459 & 89.6643 \\
\hline
\end{tabular}

Table S1. Calculated lattice constant parameters of $\mathrm{A}_{2} \mathrm{PbI}_{4}, \mathrm{~A}_{2} \mathrm{MAPb}_{2} \mathrm{I}_{7}$ and $\mathrm{A}_{2} \mathrm{MA}_{2} \mathrm{~Pb}_{3} \mathrm{I}_{10}\left(\mathrm{~A}=\mathrm{PEA}\right.$, FPEA, and $\left.\mathrm{F}_{3} \mathrm{MPEA}\right)$. 


\begin{tabular}{ccccc}
\hline Perovskite components & $A_{1}$ & $\tau_{1} / \mathrm{s}$ & $A_{2}$ & $\tau_{2} / \mathrm{s}$ \\
\hline Control & 1533.1 & 3.9364 & 1640.6 & 200.86 \\
PEAI-modified & 8105.3 & 1.4370 & 2042.8 & 162.88 \\
FPEAI-modified & 4458.2 & 5.4233 & 1830.8 & 67.538 \\
F $_{3}$ MPEAI-modified & 18081.2 & 1.2830 & 1614.1 & 61.197 \\
\hline
\end{tabular}

Table S2. Parameters derived from time-resolved photoluminescence (TRPL) results, where $A_{1}, A_{2}$ are time-independent coefficient of amplitude fraction, $\tau_{1}, \tau_{2}$ are fast decay time and slow decay time, respectively. 


\begin{tabular}{ccc}
\hline Heterojunction components & Work function difference/eV & Charge transfer/e \\
\hline$(\mathrm{PEA})_{2} \mathrm{PbI}_{4} / \mathrm{MAPbI}_{3}$ & 0.05 & 0.918 \\
$(\mathrm{FPEA})_{2} \mathrm{PbI}_{4} / \mathrm{MAPbI}_{3}$ & 0.40 & 0.925 \\
$\left(\mathrm{~F}_{3} \mathrm{MPEA}\right)_{2} \mathrm{PbI}_{4} / \mathrm{MAPbI}_{3}$ & 0.71 & 0.938 \\
$(\mathrm{PEA})_{2} \mathrm{MAPb}_{2} \mathrm{I}_{7} / \mathrm{MAPbI}_{3}$ & 0.03 & 0.922 \\
$(\mathrm{FPEA})_{2} \mathrm{MAPb}_{2} \mathrm{I}_{7} / \mathrm{MAPbI}_{3}$ & 0.37 & 0.930 \\
$\left(\mathrm{~F}_{3} \mathrm{MPEA}\right)_{2} \mathrm{MAPb}_{2} \mathrm{I}_{7} / \mathrm{MAPb}_{3}$ & 0.67 & 0.941 \\
$(\mathrm{PEA})_{2} \mathrm{MA}_{2} \mathrm{~Pb}_{3} \mathrm{PbI}_{10} / \mathrm{MAPbI}_{3}$ & 0.03 & 0.940 \\
$(\mathrm{FPEA})_{2} \mathrm{MA}_{2} \mathrm{~Pb}_{3} \mathrm{PbI}_{10} / \mathrm{MAPbI}_{3}$ & 0.37 & 0.932 \\
$\left(\mathrm{~F}_{3} \mathrm{MPEA}\right)_{2} \mathrm{MA}_{2} \mathrm{~Pb}_{3} \mathrm{PbI}_{10} / \mathrm{MAPbI}_{3}$ & 0.66 & 0.943 \\
\hline
\end{tabular}

Table S3. Calculated work function difference and charge transfer between $2 \mathrm{D} / 3 \mathrm{D}$ heterojunctions and $\mathrm{MAPbI}_{3}$, with 2D perovskites $(\mathrm{n}=1,2$ and 3$)$ formed by PEA, FPEA and $\mathrm{F}_{3} \mathrm{MPEA}$. 


\begin{tabular}{|c|c|c|c|c|c|}
\hline Perovskite components & $\begin{array}{c}\text { Polar } \\
\text { molecule }\end{array}$ & $\begin{array}{c}\text { Location of } \\
\text { EDM }\end{array}$ & $\begin{array}{l}\text { PCE } \\
(\%)\end{array}$ & $\begin{array}{c}\text { Published } \\
\text { year }\end{array}$ & Ref \\
\hline $\mathrm{CH}_{3} \mathrm{NH}_{3} \mathrm{I}_{3-\mathrm{x}} \mathrm{Cl}_{\mathrm{x}}$ & PEIE & Perovskite/ITO & 15.0 & 2016 & 1 \\
\hline $\mathrm{Cs}_{0.05} \mathrm{FA}_{0.79} \mathrm{MA}_{0.16} \mathrm{PbI}_{2.49} \mathrm{Br}_{0.51}$ & HS-Ph-CN & Perovskite/HTL & 20.2 & 2018 & 2 \\
\hline \multirow[t]{2}{*}{$\mathrm{MAPbI}_{3}$} & PFN\& & Al/ETL\& & 19.4 & 2018 & 3 \\
\hline & P-PFP-O & ITO/HTL & & & \\
\hline $\mathrm{MAPbI}_{3}$ & PPEA & Perovskite/ETL & 20.9 & 2019 & 4 \\
\hline$\left[(\mathrm{BA})_{0.94}\left(\mathrm{~F}_{3} \mathrm{EA}\right)_{0.06}\right]_{2}(\mathrm{MA})_{3} \mathrm{~Pb}_{4} \mathrm{I}_{13}$ & $\mathrm{~F}_{3} \mathrm{EA}$ & 2D perovskite & 12.5 & 2019 & 5 \\
\hline $\mathrm{MAPbI}_{3}$ & $\pi$-PFEs & $\mathrm{Ag} / \mathrm{ETL}$ & 18.5 & 2019 & 6 \\
\hline $\mathrm{MAPbI}_{3}$ & TIPD & Perovskite/ETL & 15.2 & 2019 & 7 \\
\hline$\left(\mathrm{FAPbI}_{3}\right)_{0.85}\left(\mathrm{MAPbBr}_{3}\right)_{0.15}$ & $\mathrm{~F}_{3} \mathrm{EA}$ & Perovskite/HTL & 19.2 & 2019 & 8 \\
\hline $\mathrm{MAPbI}_{3}$ & $\mathrm{TZ}$ & HTL & 18.0 & 2029 & 9 \\
\hline $\mathrm{MAPbI}_{3}$ & TMBA & Perovskite/ETL & 13.8 & 2020 & 10 \\
\hline$\left(\mathrm{FA}_{\mathrm{x}} \mathrm{MA}_{1-\mathrm{x}}\right) \mathrm{Pb}\left(\mathrm{I}_{\mathrm{y}} \mathrm{Br}_{1-\mathrm{y}}\right)_{3}$ & $\mathrm{~F}_{3} \mathrm{MPEA}$ & Perovskite/HTL & 22.4 & & $\begin{array}{l}\text { This } \\
\text { work }\end{array}$ \\
\hline
\end{tabular}

Table S4. Performances of PSC devices, with various polar molecule modification and location of electric dipole moments. 


\section{References}

1. Song, S.; Moon, B. J.; Hörantner, M. T.; Lim, J.; Kang, G.; Park, M.; Kim, J. Y.; Snaith, H. J.; Park, T. Interfacial Electron Accumulation for Efficient Homo-Junction Perovskite Solar Cells. Nano Energy 2016, 28, 269-276.

2. Lu, J. F.; Lin, X. F.; Jiao, X. C.; Gengenbach, T.; Scully, A. D.; Jiang, L. C.; Tan, B.; Sun, J. S.; Li, B.; Pai, N., et al. Interfacial Benzenethiol Modification Facilitates Charge Transfer and Improves Stability of Cm-Sized Metal Halide Perovskite Solar Cells with up to 20\% Efficiency. Energy Environ. Sci. 2018, 11, 1880-1889.

3. Lee, J. H.; Kim, J.; Kim, G.; Shin, D.; Jeong, S. Y.; Lee, J.; Hong, S.; Choi, J. W.; Lee, C. L.; Kim, H., et al. Introducing Paired Electric Dipole Layers for Efficient and Reproducible Perovskite Solar Cells. Energy Environ. Sci. 2018, 11, 1742-1751.

4. Dong, H.; Xi, J.; Zuo, L.; Li, J.; Yang, Y.; Wang, D.; Yu, Y.; Ma, L.; Ran, C.; Gao, W., et al. Conjugated Molecules "Bridge": Functional Ligand toward Highly Efficient and Long-Term Stable Perovskite Solar Cell. Adv. Funct. Mater. 2019, 29, 1808119.

5. Tan, S.; Zhou, N.; Chen, Y.; Li, L.; Liu, G.; Liu, P.; Zhu, C.; Lu, J.; Sun, W.; Chen, Q., et al. Effect of High Dipole Moment Cation on Layered 2d Organic-Inorganic Halide Perovskite Solar Cells. Adv. Energy. Mater. 2019, 9, 1803024.

6. An, Q. Z.; Sun, Q.; Weu, A.; Becker-Koch, D.; Paulus, F.; Arndt, S.; Stuck, F.; Hashmi, A. S. K.; Tessler, N.; Vaynzof, Y. Enhancing the Open-Circuit Voltage of Perovskite Solar Cells by up to $120 \mathrm{Mv}$ Using Pi-Extended Phosphoniumfluorene Electrolytes as Hole Blocking Layers. Adv. Energy Mater. 2019, 9, 9 .

7. Weng, Y. J.; Shen, Z. T.; Guo, M. X.; Wu, F.; Li, F. M.; Zhu, L. X.; Ling, L. Y.; Chen, C. Electric Dipole Moment-Assisted Charge Extraction and Effective Defect Passivation in Perovskite Solar Cells by Depositing a Pcbm:Tipd Blend Film on a Ch3nh3pbi3 Layer. J. Mater. Chem. C 2019, 7, 1155911568.

8. Xu, H. F.; Sun, Y. H.; Zheng, H. Y.; Liu, G. Z.; Xu, X. X.; Xu, S. D.; Zhang, L. Y.; Chen, X. J.; Pan, X. High-Performance and Moisture-Stable Perovskite Solar Cells with a 2d Modified Layer Via Introducing a High Dipole Moment Cation. J. Mater. Chem. C 2019, 7, 15276-15284.

9. Butscher, J. F.; Intorp, S.; Kress, J.; An, Q.; Hofstetter, Y. J.; Hippchen, N.; Paulus, F.; Bunz, U. H. F.; Tessler, N.; Vaynzof, Y. Enhancing the Open-Circuit Voltage of Perovskite Solar Cells by Embedding Molecular Dipoles within Their Hole-Blocking Layer. ACS Appl. Mater. Interfaces 2020, 12, 3572-3579.

10. Han, J.; Kwon, H.; Kim, E.; Kim, D. W.; Son, H. J.; Kim, D. H. Interfacial Engineering of a Zno Electron Transporting Layer Using Self-Assembled Monolayers for High Performance and Stable Perovskite Solar Cells. J. Mater. Chem. A 2020, 8, 2105-2113. 